\title{
Effect of cellular mass on chondrogenic differentiation during embryoid body formation
}

\author{
MICHAŁ STEFAN LACH ${ }^{1-3^{*}}$, KATARZYNA KULCENTY ${ }^{1,3^{*}}$, KAROLINA JANKOWSKA ${ }^{1}$, \\ TOMASZ TRZECIAK ${ }^{4}$, MAGDALENA RICHTER $^{4}$ and WIKTORIA MARIA SUCHORSKA ${ }^{1,3}$ \\ ${ }^{1}$ Radiobiology Lab, Greater Poland Cancer Centre, 61-866 Poznan; ${ }^{2}$ Postgraduate School of Molecular Medicine, \\ Warsaw University of Medical Sciences, 02-091 Warsaw; ${ }^{3}$ Department of Electroradiology, \\ Poznan University of Medical Sciences, 61-866 Poznan; ${ }^{4}$ Department of Orthopaedics and Traumatology, \\ Poznan University of Medical Sciences, 61-545 Poznan, Poland
}

Received February 8, 2018; Accepted July 3, 2018

DOI: $10.3892 / \mathrm{mmr} .2018 .9272$

\begin{abstract}
One approach to cell differentiation is to use the natural capacity of pluripotent stem cells to form three germ layers via embryoid bodies (EB). However, unification of this process during in vitro culture remains challenging and many microenvironmental factors including the number of cells in the culture can influence differentiation patterns. The number of cells serves a crucial role as it determines access to nutrients, the distribution of oxygen concentration and cellular interactions, all of which influence the fate of the differentiated cells. The influence of EBs derived from human pluripotent cells on the chondrogenic potential of such cells is not well understood. For this reason, the present study sought to determine the effect of varying amounts of cells on the properties of EBs derived from human embryonic stem cells (BG01V cell line). In the present study, 500-2,000 cells per well were cultivated from 5 to 15 days in suspension cell culture. Expression of pluripotency genes and germ layer markers were evaluated in order to determine the EBs with the greatest and least mesodermal properties. Genes associated with pluripotency and chondrogenesis were also evaluated to assess the influence of suspension culture duration and EB size on chondrogenic differentiation. Immunofluorescence staining for pluripotent and chondrocyte-associated proteins confirmed successful differentiation into chondrocyte-like cells. Alcian blue staining confirmed deposition of proteoglycans. These results suggested that EBs formed in 500-cell wells possess the highest mesodermal and prochondrogenic properties.
\end{abstract}

Correspondence to: Mr. Michał Stefan Lach, Radiobiology Lab, Greater Poland Cancer Centre, Garbary 15 Street, 61-866 Poznan, Poland

E-mail: lach.micha189@gmail.com

${ }^{*}$ Contributed equally

Key words: chondrogenic differentiation, chondrocytes, human embryonic stem cells, embryoid bodies
Differentiation of EBs into chondrocytes on day 5 in 500-cell wells was more efficient than in that observed in larger and older EBs.

\section{Introduction}

The rising incidence of osteoarthritis in developed countries has caused numerous socio-economic problems (1-3). For this reason, more effective treatments are needed. One approach to treating the damaged cartilage in osteoarthritis is matrix-induced autologous chondrocyte implantation (MACI), a process that requires millions of cells (4). However, an important drawback of MACI is that chondrocytes propagated in an in vitro culture tend to lose their primary function and phenotype in a process known as dedifferentiation. Thus, during MACI, type I collagen production increases relative to type II collagen, which is uncommon in hyaline cartilage chondrocytes (5). To overcome this drawback, several studies have differentiated multipotent and pluripotent stem cell populations into chondrocyte-like cells. Multipotent stem cells, such as mesenchymal stem cells (MSCs), can be easily obtained from numerous different sources in the body, including fat and bone marrow. However, the low concentration of MSCs in the general cell population requires propagation in an in vitro culture. In addition, MSCs may not be feasible for the treatment of degenerative diseases because both the number of these cells and their proliferative capacity decrease with age (6-8). For this reason, other cell sources, such as pluripotent stem cells which have unlimited proliferative and self-renewal ability would seem to be a better option for therapeutic purposes $(9,10)$. However, the use of pluripotent stem cells, especially human embryonic stem cells (hESCs), is controversial and may raise ethical issues. These objections can be overcome by using induced pluripotent stem cells (iPSCs), although such cells have several limitations, including safety issues related to their tumorigenic potential and the unknown efficiency of differentiation into chondrocytes. Additionally, some studies suggest that there is an important difference between iPSCs and hESCs at the molecular level (11). Other disadvantages of iPSCs are the high cost of culture and the low reprogramming efficiency $(11,12)$. 
Various chondrogenic differentiation protocols have been described in recent years, including high density mass, micromass (13), monolayer culture (14), and embryoid bodies (EB) formation which is probably the most common protocol $(15,16)$. The EB-based protocol takes advantage of the natural ability of pluripotent stem cells to form three germ layers. EBs can be derived through a variety of methods, including suspension culture, hanging-drops, or size-defined wells $(17,18)$. However, the heterogeneous size of EBs affects their microenvironment because oxygen levels, growth factors, and nutrient concentration all vary depending on the EB depth in the culture. Moreover, changes in these factors could affect the spontaneous differentiation process (19). Besides the physical properties, the number of cells used in EB-formation also influences signalling pathways. Apart from size, one of the potential regulators of EB differentiation is the non-canonical WNT pathway, which directly impacts the differentiation of cells into specific germ layers. Hwang et al (20) showed that WNT5a (a regulator of vasculogenesis) was elevated in $150 \mu \mathrm{m}$ diameter hydrogel wells used to form size-defined EBs, whereas EBs formed in $450 \mu \mathrm{m}$ wells presented increased expression of WNT11 (a regulator of cardiomyogenic cells). Nevertheless, at present, the influence of the size of EBs derived from human pluripotent cells on chondrogenic fate remains poorly understood due to a lack of data.

In this context, the aim of this study was to demonstrate how the number of cells used for EB formation could improve differentiation protocols used to create chondrocyte-like cells for regenerative purposes and/or for the study of chondrogenesis. In addition, we sought to determine the effect of cell colony size and culture time on the spontaneous differentiation of hESCs and further chondrogenic differentiation.

\section{Materials and methods}

HESC culture. The BG01V hESC line (American Type Culture Collection, Manassas, VA; USA) was cultured in mitomycin-C-treated mouse embryonic fibroblasts (MEFs; passage 3; Sigma-Aldrich; Merck KGaA, Darmstadt, Germany) seeded on a culture dish previously coated with Matrigel ${ }^{\mathrm{TM}}$ (Corning Incorporated, Corning, NY, USA). The hESCs were cultured in DMEM/F12 (Merck Millipore, Germany) supplemented with $20 \%$ KnockOut $^{\mathrm{TM}}$ Serum Replacement (KnockOut ${ }^{\mathrm{TM}}$ SR, Thermo Fisher Scientific Inc., MA, USA), $1 \%$ penicillin-streptomycin (Sigma-Aldrich; Merck KGaA), $1 \mathrm{mM}$ non-essential amino acid (NEAA; Sigma-Aldrich; Merck KGaA), 0.2 mM 2-mercaptoethanol (Sigma-Aldrich; Merck KGaA), and $10 \mathrm{ng} / \mathrm{ml}$ basic fibroblast growth factor (bFGF) (Sigma-Aldrich; Merck KGaA). Cells were cultured at $37^{\circ} \mathrm{C}$ in a humidified atmosphere containing $5 \% \mathrm{CO}_{2}$ and $95 \%$ air.

Embryoid body formation. The hESC colonies were dissociated enzymatically with $0.1 \%$ EDTA/Trypsin (Sigma-Aldrich; Merck KGaA). Then the cells were counted and seeded onto 96-well plate dedicated for suspension cell culture (inertGrade $^{\mathrm{TM}}$; BRAND GMBH + CO KG, Wertheim, Germany) in varying cell numbers $(500,1,000,1,500$, and 2,000 cells per well) (Fig. 1) with the addition of $10 \mu \mathrm{M}$ ROCK-inhibitor
Y-27632 (Sigma-Aldrich; Merck KGaA). Next, cells were left for spontaneous aggregation for 15 days. The medium (hESC medium without bFGF) was changed every second day.

Measurement of EB size and homogeneity. The diameter of the EBs was measured with a tool provided by the manufacturer of the IS-OptaView programme (Opta-Tech, Warszawa, Poland) which was designed for use with the inverted-microscope MF-100F (Opta-Tech). EB size was measured on the 5th, 10th and 15 th day of culture ( $\mathrm{n}=25$ for each variant and day). Homogeneity of the formed EBs $(n=2,300)$ was also evaluated.

Chondrogenic differentiation of EBs. EBs $(\mathrm{n}=20)$ were transferred onto 6-well plates coated with Matrigel ${ }^{\text {TM }}$ (Corning Incorporated). After $24 \mathrm{~h}$, the medium was replaced with a chondrogenic medium (ChM) (day 0) composed of DMEM/F12 (Sigma-Aldrich; Merck KGaA), 2\% KnockOut ${ }^{\mathrm{TM}}$ SR (Thermo Fisher Scientific, Inc., Waltham, MA, USA), $1 \mathrm{mM}$ sodium pyruvate (Biowest, Nuaillé, France), $10^{-7} \mathrm{M}$ dexamethasone, $50 \mu \mathrm{M}$ ascorbic acid, $50 \mu \mathrm{M}$ L-proline, $1 \%$ penicillin-streptomycin (all provided by Sigma-Aldrich; Merck KGaA), $1 \%$ ITS+ premix (Corning Incorporated), and $10 \mathrm{ng} / \mathrm{ml}$ TGF- $\beta 3$ (ImmunoTools, Friesoythe, Germany). The EBs were cultured for 21 days and the medium was changed every second day.

Total RNA isolation, cDNA synthesis and reverse transcription-quantitative polymerase chain reaction $(R T-q P C R)$. To isolate the total RNA, 25 EBs from suspension culture or EBs after chondrogenic differentiation were collected and suspended in TRI Reagent (Sigma-Aldrich; Merck $\mathrm{KGaA}$ ). The procedure was performed according to the manufacturer's instructions. Then $1 \mu \mathrm{g}$ of total RNA was used for cDNA synthesis with iScript ${ }^{\mathrm{TM}}$ cDNA synthesis kit (BioRad Laboratories, Inc., Hercules, CA, USA) according to the manufacturer's specifications. Next, the gene expression profile was evaluated by RT-qPCR by applying FAM-labelled probes (Roche Applied Science, Penzberg, Germany). The thermocycling conditions were as follows: Preincubation for $10 \mathrm{~min}$ at $95^{\circ} \mathrm{C}$, followed by the amplification step (denaturation at $95^{\circ} \mathrm{C}$ for $10 \mathrm{sec}$, annealing at $60^{\circ} \mathrm{C}$ for $30 \mathrm{sec}$ and extension at $72^{\circ} \mathrm{C}$ for $1 \mathrm{sec}$ ), and then cooling at $40^{\circ} \mathrm{C}$ for $30 \mathrm{sec}$. Primers and specific probes were designed using the online Roche Universal ProbeLibrary software. All primers used in this study are listed in Table I. Gene expression was estimated by the $2^{-\Delta \Delta C a}$ method (21). Glyceraldehyde 3-phosphate dehydrogenase (GAPDH) was used as the reference gene. The data were normalized to the expression level of the control cell populations of HC-402-05a (Human Chondrocytes; Cell Applications Inc., San Diego, CA, USA) or the BG01V cell line.

Alcian blue staining. Alcian blue staining was performed to confirm the deposition of proteoglycans (a marker of cartilage formation). Firstly, EBs $(n=6)$ were transferred into a 12-well plate coated with Matrigel ${ }^{\mathrm{TM}}$. After 21 days of differentiation, cells were fixed with 4\% paraformaldehyde (Sigma-Aldrich; Merck KGaA) for $20 \mathrm{~min}$. After three washes in phosphate buffered saline (PBS; Biowest), cells were incubated with $1 \%$ Alcian blue (Sigma-Aldrich; Merck KGaA) with $0.1 \mathrm{~N}$ $\mathrm{HCl}$ (POCH S.A., Gliwice, Poland) $(\mathrm{pH}=1.0)$. Next, stained cells were rinsed in $0.1 \mathrm{~N} \mathrm{HCl}$ and washed twice in PBS. 
A

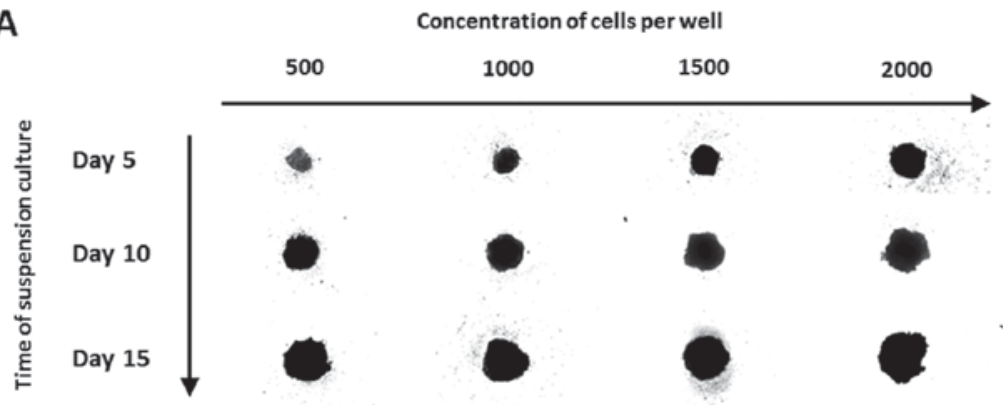

B Diameter of embryoid bodies during suspension culture

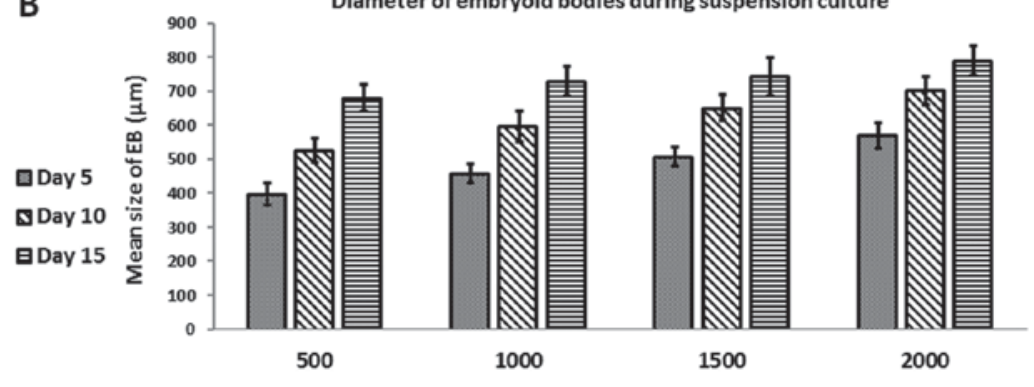

Amount of cells per well

Figure 1. Growth is faster in EBs formed in wells with a lower cell concentration. (A) EB were evaluated microscopically during the 15 day suspension culture period. This analysis revealed that mass growth was increased in smaller EBs when compared with larger EBs. (B) The EBs were measured at days 5 , 10 and 15 of the suspension culture. Data are presented as the mean \pm standard deviation of spherical EBs measurements ( $\mathrm{n}=25$ ). EBs, embryoid bodies.

Then, pictures were taken under 40x magnification using a tool provided by the manufacturer of the IS-OptaView programme (Opta-Tech) which was designed for use with the inverted-microscope MF-100F (Opta-Tech). All steps were performed at room temperature. The HC-402-05a and the BG01V cell lines were used as controls.

Indirect immunofluorescence. Indirect immunofluorescence staining was performed to confirm the presence of chondrogenic marker expression and the absence of pluripotent cells after differentiation. Cells were washed with phosphate-buffered saline (Biowest) and then fixed with cooled methanol for $20 \mathrm{~min}$ at $-20^{\circ} \mathrm{C}$. Next, the cells were blocked with PBS containing $1 \%$ bovine serum albumin (BSA; Biowest) and $0.5 \%$ Tween (POCH, Poland). Cells were then incubated for $1 \mathrm{~h}$ at $37^{\circ} \mathrm{C}$ with primary antibodies diluted in PBS containing $1 \%$ BSA at optimised dilution (Table II). After the washes, a secondary antibody anti-rabbit conjugated with Alexa Fluor 488 or anti-mouse IgG conjugated with Alexa Fluor 488 (in all cases, diluted 1:500; Jackson ImmunoResearch Laboratories, Inc., West Grove, PA, USA) were added and incubated for $1 \mathrm{~h}$ at $37^{\circ} \mathrm{C}$. After these washes, the nuclei were stained with 4',6-Diamidino-2-phenylindole dihydrochloride (DAPI) at 1:10,000 dilution (Sigma-Aldrich; Merck KGaA). Images were taken using the IS-Opta-View application from the fluorescence module of the MW-100F inverted microscope (Opta-Tech). A hESC cell line and HC-402-05a were used as controls. To indicate the specific binding of primary antibodies, the control reaction with $1 \%$ BSA was performed (data not shown).

Statistical analysis. All experiments were performed in triplicate and the data are presented as the mean \pm standard deviation of three replicates. The results were compared using two-way analysis of variance with a Holm-Sidak post-hoc test, and unpaired tailed Student t-test (GraphPad Prism version 6.01; GraphPad Software, Inc., La Jolla, CA, USA). $\mathrm{P}<0.05$ was considered to indicate a statistically significant difference.

\section{Results}

Measurement of $E B$ diameter and efficacy of $E B$ formation. To assess the influence of EB size on chondrogenic differentiation, hESC cells (500, 1,000, 1,500 and 2,000 per well) were seeded and cultured for 15 days. During suspension culture, the EBs were assessed by microscope to determine the size (diameter) and homogeneity (formation of single, round sphere) (Fig. 1A and B). Most (78.1\%) of the EBs were homogenous (Table III). EBs formed in wells seeded with 500 cells measured a mean of $397.92 \pm 31.91 \mu \mathrm{m}$ in diameter at day $5,526.06 \pm 36.46 \mu \mathrm{m}$ at day 10 , and $680.33 \pm 38.46 \mu \mathrm{m}$ at the final cell count (day 15, the end of the suspension culture). The corresponding values for wells seeded with 1,000 cells were as follows: $457.85 \pm 28.46,596.17 \pm 44.5$, and $728.1 \pm 42.94 \mu \mathrm{m}$ on days 5, 10 and 15, respectively. In wells with 1,500 cells, the values on days 5,10 and 15 were: $508 \pm 28.31,651.31 \pm 38.49$, and $743.04 \pm 56.96 \mu \mathrm{m}$, respectively. The largest EBs were formed in wells seeded with 2,000 cells, as follows: $568.91 \pm 36.02$, $702.36 \pm 41.27$, and $789.06 \pm 42.30 \mu \mathrm{m}$. These changes in EB diameters indicate increased cell proliferation in the smaller EBs formed in 500-cell wells vs. those with 2,000 cells.

The number of cells used for EB formation affects the spontaneous differentiation of these cells into three germ layers. Gene expression was estimated on days 5, 10 and 15 of the EB suspension culture procedure. The following markers were 
Table I. Primers used in the present study.

\begin{tabular}{|c|c|c|c|}
\hline Target mRNA & Forward sequence (5'-3') & Reverse sequence (5'-3') & Reference sequence \\
\hline ACAN & CGTGAATCAGAATCAACTGCTG & GTGTCCCTCTGTCTCCTTGC & NM_013227.3 \\
\hline ACTA2 (SMA) & CTGTTCCAGCCATCCTTCAT & TCATGATGCTGTTGTAGGTGGT & NM_001141945.1 \\
\hline BRACHYURY (T) & GATGATCGTGACCAAGAACG & CTTCCAGCGGTGGTTGTC & NM_003181.2 \\
\hline CD44 & AGCCTACTGCAAATCCAAACA & GAAGCTCTGAGAATTACTCTGCTG & NM_000610.3 \\
\hline $\mathrm{CDH} 2$ & ATCCGACGAATGGATGAAAG & CTGTGGGGTCATTGTCAGC & NM_001792.3 \\
\hline COL1A2 & GGCAGTGATGGAAGTGTGG & CCAACAGCTCCAATTTCACC & NM_000089.3 \\
\hline COL2A1 & TTCTGGAGACCAAGGTGCTT & TTCCATTAGCACCATCTTTGC & NM_001844.4 \\
\hline FGFR3 & AGAGGCCCACCTTCAAGC & CGACAGGTCCAGGTACTCGT & NM_000142.4 \\
\hline FOXA2 & CGCCCTACTCGTACATCTCG & AGCGTCAGCATCTTGTTGG & NM_021784.4 \\
\hline MIXL1 & AAGCGCACGTCTTTCAGC & GCCTGTTCTGGAACCATACCT & NM_031944.1 \\
\hline NANOG & ATGCCTCACACGGAGACTGT & AAGTGGGTTGTTTGCCTTTG & NM_024865.2 \\
\hline NCAM1 & CGACCATCCACCTCAAAGTC & CGGAGGCTTCACAGGTAAGA & NM_000615.6 \\
\hline PAX6 & GGCACACACACATTAACACACTT & GGTGTGTGAGAGCAATTCTCAG & NM_000280.3 \\
\hline SOX2 & TGCCTCTTTAAGACTAGGACTGAGA & GCCGCCGATGATTGTTATTA & NM_003106.3 \\
\hline SOX9 & CTCGCCACACTCCTCCTC & CGCTTCAGGTCAGCCTTG & NM_000346.3 \\
\hline TNC & GCTCAAAGCAGCCACTCATT & CCCATATCTGGAACCTCCTCT & NM_002160.3 \\
\hline VIM & GGGACCTCTACGAGGAGGAG & CTTTGTCGTTGGTTAGCTGGT & NM_003380.3 \\
\hline
\end{tabular}

SOX, sex determining region Y-box; MIXL1, mix paired-like homeobox; SMA, $\alpha$-smooth muscle actin; FOXA2, forkhead box protein A2; VIM, vimentin; PAX6, paired box 6; FGFR3, fibroblast growth factor receptor 3; CD44, cluster of differentiation 44; COL, collagen; ACAN, aggrecan; TNC, tenascin C; CDH2, cadherin 2; NCAM, neural cell adhesion molecule.

evaluated to assess spontaneous differentiation (Fig. 2A-H): pluripotency (NANOG, SRY [sex determining region Y]-box 2 (SOX2)], primitive streak mesoendoderm [MIXL, Brachyury (T)], ectoderm (VIM, PAX6), endoderm [forkhead box protein A2 (FOXA2)], and mesenchyme [ $\alpha$-smooth muscle actin $(\alpha$-SMA)]. Expression of the pluripotency markers NANOG (Fig. 2A) and SOX2 (Fig. 2B) decreased in all well variants compared to controls. EBs expressing favourable pluripotent markers were not observed in any of the variants. Expression of the mesodermal marker Brachyury (Fig. 2C) was most significant on day 15 in the 500 and 1,000 cell wells, indicating the pro-mesodermal potential of those EBs compared to controls and the other variants (e.g., 1,500 and 2,000 cell wells) at other time points. Surprisingly, the mesodermal marker MIXL1 (Fig. 2D) was more highly expressed in EBs formed in 500 and 1,000 cell wells at day 15 vs. controls and other variants. No differences in expression of the mesenchymal marker SMA (Fig. 2E) were observed in EBs compared to hESCs. During suspension culture, SMA expression increased in all cell colony variants, with the highest expression observed at day 10 in EBs cultured in 2,000 cell cultures compared to the other culture variants. Compared to controls, expression of the endoderm marker gene FOXA2 (Fig. 2F) was lower on days 5 and 10 but higher on day 15. Expression of this marker increased throughout the suspension culture period in all EB populations. A significant increase was observed at day 10 in the 2,000-cell wells and at day 15 in the 1,000-cell wells. Compared to BG01V, expression of the ectoderm marker VIM (Fig. 2G) decreased notably in all variants on days 5, 10, and 15 . The only exceptions were in the 500 and 1,000 cell EB: On day 15, VIM expression in EBs was similar to the control. Similarly, the 500 and 1,000 cell variants presented the highest expression of VIM compared to the 1,500 and 2,000 cell cultures assessed earlier in the suspension culture. PAX6 (Fig. 2H) expression increased over time, achieving levels comparable to controls at days 10 and 15. PAX6 expression was highest in the 500 and 1,000 cell variants on day 10 , and in the 500 cell variant on day 15 compared to the other groups.

The influence of EB size on chondrogenic differentiation. Based on the gene expression of EBs evaluated during suspension culture, EBs formed in the larger $(2,000$ cell) colonies presented lower levels of mesodermal expression whereas the EBs with the greatest mesodermal expression were formed in the smaller (500 cell) wells. EBs were collected on days 5 and 15 to test the hypothesis that prolonged time in suspension culture would improve differentiation into chondrocytes due to the increasing expression of mesodermal markers over time. To perform this test, we used a serum-free chondrogenic medium supplemented with TGF- $\beta 3$. After differentiation, gene expression analysis was performed to identify genes associated with pluripotency (NANOG and SOX2); mesoderm (T); chondrocytes [SOX9, FGFR3, CD44, type I collagen (COL1A2), COL2A1 and ACAN], and mesenchymal condensation [Neuronal Cell Adhesion Molecule (NCAM), N-cadherin (CDH2) and tenascin-C (TNC)] (Figs. 3 and 4).

Compared to hESC cells, NANOG and SOX2 expression decreased significantly in the differentiated EBs (Fig. 3A and B). Among the various cell colony variants, EBs differentiated in 2,000-cell wells collected on day 15 presented the lowest expression of pluripotency markers; by contrast, expression 
Table II. List of primary and secondary antibodies used in the present study.

A, Primary antibodies

\begin{tabular}{llll}
\hline Antibody & Host/isotype & \multicolumn{1}{c}{ Supplier (cat. no.) } \\
\hline NANOG & Mouse/IgG1 & $\begin{array}{l}\text { BD Pharmingen; BD Biosciences, } \\
\text { San Jose, CA, USA (560482) }\end{array}$ \\
SOX9 & Rabbit/IgG & Abcam, Cambridge, UK (ab71762) & $1: 50$ \\
Type II collagen & Rabbit/IgG & Abcam (ab34712) & $1: 50$ \\
Chondroitin sulphate & Mouse/IgM & Abcam (ab11570) & $1: 100$ \\
\hline
\end{tabular}

B, Secondary antibodies

\begin{tabular}{lll}
\hline Antibody & Host/isotype & \multicolumn{1}{c}{ Supplier (cat. no.) } \\
\hline $\begin{array}{l}\text { Anti-mouse IgG conjugated } \\
\text { with Alexa Fluor 488 }\end{array}$ & Donkey/IgG & Jackson ImmunoResearch Laboratories, \\
$\begin{array}{l}\text { Anti-rabbit IgG conjugated } \\
\text { with Alexa Fluor 488 }\end{array}$ & Donkey/IgG & Jackson ImmunoResearch laboratories, Inc., \\
$\begin{array}{l}\text { Anti-mouse IgM conjugated } \\
\text { with Alexa Fluor 488 }\end{array}$ & Donkey/IgG & $\begin{array}{l}\text { Jackson ImmunoResearch laboratories, Inc., } \\
\text { (715-545-152) }\end{array}$ \\
\hline
\end{tabular}

SOX9, sex determining region Y-box 9; Ig, immunoglobulin.

Table III. Efficiency of homogeneous formation of spherical EBs in BRAND inertGRADE ${ }^{\mathrm{TM}}$ 96-well plates.

\begin{tabular}{lc} 
Feature of EBs per well $(\mathrm{n}=2,300)$ & Percentage $(\%)$ \\
\hline Homogeneous (single spherical EB) & 78.09 \\
Heterogeneous (double-triple-spherical, & 21.91 \\
elongated EBs) &
\end{tabular}

EBs, embryoid bodies.

of T (Fig. 3C) in differentiated EB cells was higher on day 5 compared to controls and compared to EBs collected on day 15. No significant differences in gene expression levels between EBs formed in 500 and 2,000 cell colonies were observed on day 5. SOX9 expression (Fig. 3D) decreased in all differentiated EBs relative to SOX9 expression in the $\mathrm{HC}-402-05$ a cell line. SOX9 expression was highest on day 5 in EBs formed in 500 cell cultures. FGFR3 (Fig. 3E) expression increased in EBs formed in 500 cell wells compared to controls, but not on day 5 in 2,000 cell-well cultures. Interestingly, FGFR3 expression was lower in differentiated EBs on day 15 vs. EBs collected on day 5. CD44 (Fig. 3F) expression was higher in EBs compared to controls, with the highest expression on day 5 in EBs formed in 2,000 cell cultures. CD44 expression in EBs formed in 500 and 2,000 cell cultures on day 5 and in EBs formed on day 15 from 500 cell colonies were similar, perhaps due to the dense matrix produced during 15 days of suspension culture.

After the differentiation process was complete, we analysed the genes related to extracellular matrix production. All differentiated EBs presented decreased expression of
COL1A2 compared to controls (Fig. 3G). However, EBs formed in 500 and 2,000 cell colonies and collected at day 5 expressed type I collagen more highly than EBs collected on day 15. COL2A1 is a marker of successful chondrogenic differentiation (Fig. 3H). The highest expression of COL2A1 was observed in EBs formed in 500 cell cultures harvested on day 5. On days 5 and 15, EBs differentiated in 500 cell cultures expressed COL2A1 more highly than EBs harvested from 2,000 cell wells. ACAN (Fig. 3I) expression a marker of chondrocytes associated with the production of proteoglycans was much lower in the differentiated EB cells compared to HC-402-05a. However, EBs collected on day 5 expressed ACAN more highly than those harvested on day 15. No significant changes in ACAN expression were observed among the various EB culture sizes collected on the same days.

The influence of EB size on cartilage nodule formation. Chondrocytes produce a specific extracellular matrix (ECM) consisting mostly of proteoglycans and collagens. Glycosaminoglycan (GAG) deposition was marked by alcian blue staining. Blue staining and more condensed cartilage-like nodules were more evident in EBs formed from 500 cell colonies compared to the 2,000 cell cultures (Fig. 4A). In EBs harvested on day 15, cartilage nodule condensation was difficult to assess because the cells had less motility due to the dense ECM formed during suspension culture. To explain the different phenotypes of these nodules and to confirm the influence of $\mathrm{EB}$ size on the mesenchymal condensation process, we estimated the expression of genes (i.e., NCAM, TNC and $\mathrm{CDH} 2$ ) associated with the condensation process (Fig. 4B). NCAM expression was higher in all of the cell colony variants vs. controls (Fig. 4B). The greatest in NCAM expression was observed on day 5 in EBs formed from 500 cell cultures. CDH2 

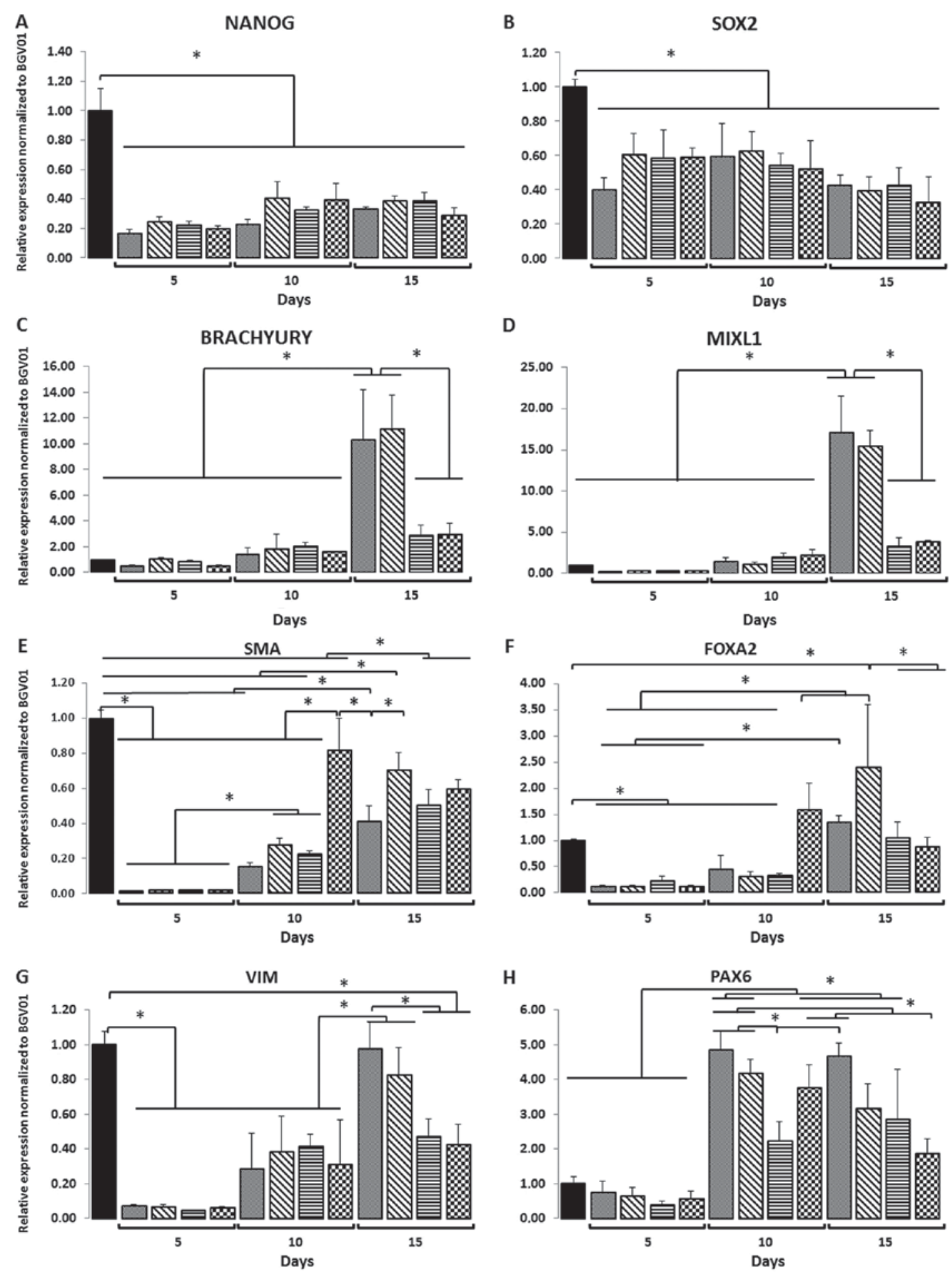

H

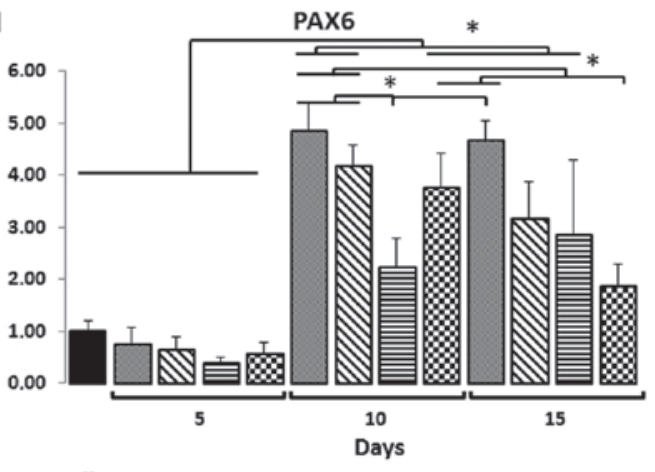

Amount of cells per well

BGV01 $\square 500$ cells 1000 cells $\mathbf{O} 1500$ cells $\mathbf{Q} 2000$ cells

Figure 2. Size of EBs and time (days) in the suspension culture affects the expression of germ layer specific genes. During suspension cell culture, the pluripotency markers, (A) NANOG and (B) SOX2, decreased among all studied variants. Gene expression analysis indicated that the mesoderm, (C) Brachyury and (D) MIXL1, germ layer was favoured in EBs formed from 500 and 1,000 cell wells when compared with the 1,500 and 2,000 cell wells at the end of suspension culture (day 15). The mesenchymal marker (E) SMA and endoderm marker (F) FOXA2 did not predominantly express in the studied variants. Gene expression analysis indicated that the ectoderm germ layer, (G) VIM and (H) PAX6, was favoured in EBs formed from 500 and 1,000 cell wells when compared with the 1,500 and 2,000 cell wells at the end of the suspension culture (day 15). The y-axis represents the relative expression of analysed genes, normalized to the BG01V cell line. Data are presented as the mean \pm standard deviation. " $\mathrm{P}<0.05$, as indicated. EBs, embryoid bodies; SOX2, sex determining region Y-box 2; MIXL1, mix paired-like homeobox; $\alpha$-SMA, $\alpha$-smooth muscle actin; FOXA2, forkhead box protein A2; VIM, vimentin; PAX6, paired box 6 .

expression was elevated in all cell culture variants compared to the HC-402-05a cell line (Fig. 4B). CDH2 expression did not differ significantly between EBs in 500 and 2,000 cell cultures collected on day 5, although $\mathrm{CDH} 2$ levels were much higher compared to EBs harvested on day 15. $\mathrm{CDH} 2$ expression was higher on day 15 in EBs formed from 500 vs. 2,000 cell 

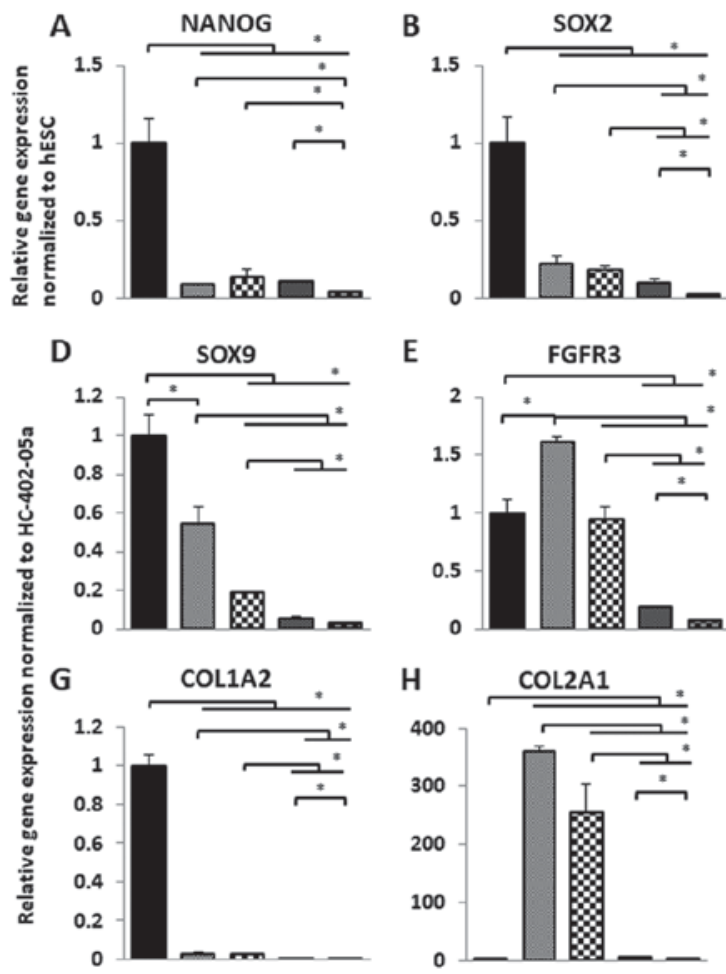

C
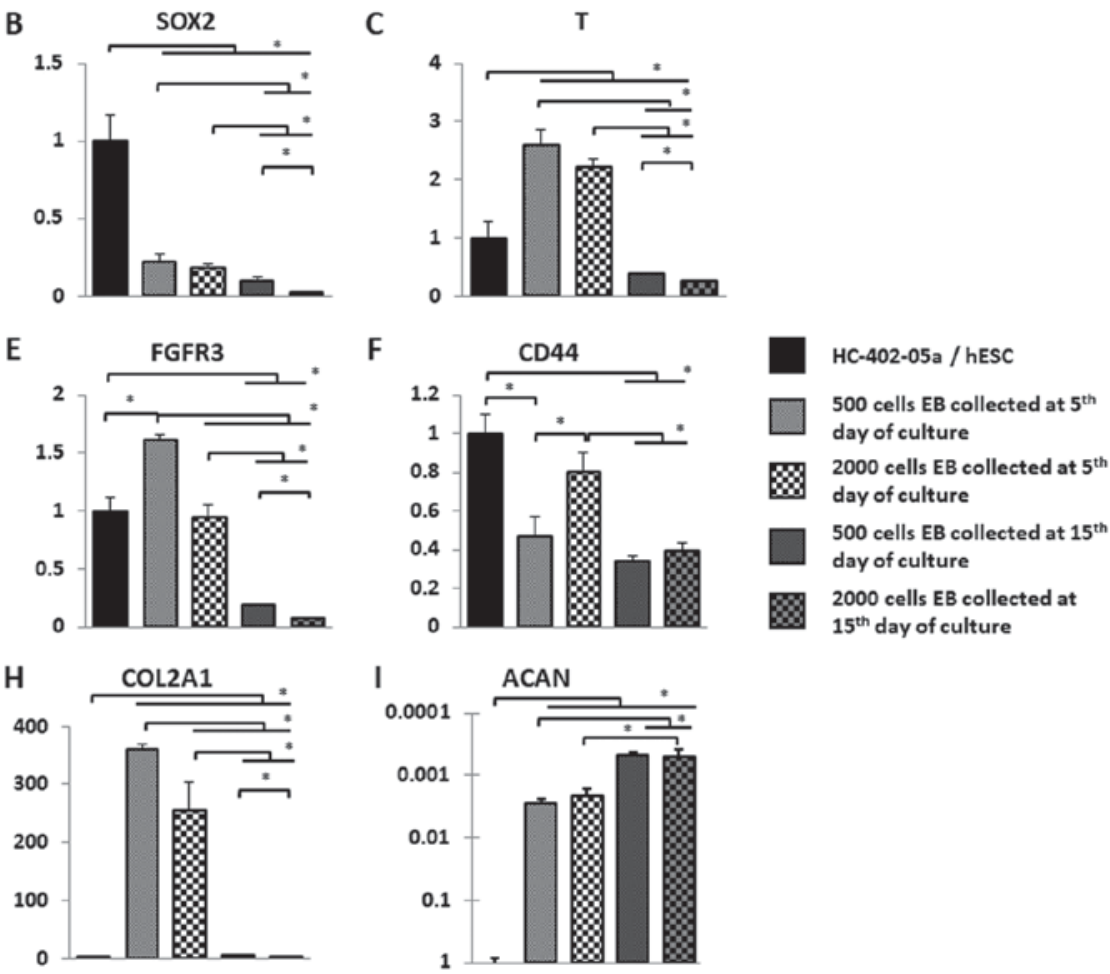

Figure 3. Smaller EBs exhibit more prochondrogenic outcomes. Following collection of EBs from suspension culture, the 3 week chondrogenic differentiation protocol was performed and gene expression was analysed. Expression of (A) NANOG and (B) SOX2 decreased in all studied variants. Expression of (C) T, (D) SOX9 and (E) FGFR3 was significantly higher in EBs formed in 500-cell wells when compared with 2,000-cell wells. (F) CD44 was significantly expressed in differentiated 2,000-cell wells EB collected on the fifth day of suspension culture. (G) The low level of COL1A2 expression was observed in all studied variants following chondrogenic differentiation. (H) Expression of COL2A1 was significantly higher in EBs formed in 500-cell wells when compared with 2,000-cell wells. (I) ACAN expression increased only in EBs collected on day 5. Overall, 15 days of suspension culture resulted in a lower expression of analysed genes in chondrogenically differentiated EBs formed from 500- and 2,000-cell wells. The y-axis represents the relative expression of analysed genes, normalized to the BG01V or HC-402-05a cell line. Data are presented as the mean \pm standard deviation. "P<0,05, as indicated. EBs, embryoid bodies; hESC, human embryonic stem cells; SOX, sex determining region Y-box; T, brachyury gene; FGFR3, fibroblast growth factor receptor 3; CD44, cluster of differentiation 44; COL, collagen; ACAN, aggrecan.

colonies. By contrast, TNC expression decreased in all studied populations compared to control cells (Fig. 4B). No significant differences were observed between TNC expression on day 5 in EBs from the 500 and 2,000 cell colonies. TNC expression was slightly higher in EBs collected on day 5 vs. day 15 . Those results indicate the presence of pre-chondrocytes and the formation of cartilage nodules in differentiated EBs harvested on days 5 and 15 of suspension culture.

Chondrocyte-like and pluripotent cells are observed in differentiated EBs. Indirect immunofluorescence staining was performed for SOX9, type II collagen, chondroitin sulphate, and NANOG to confirm the presence of proteins related to chondrocyte-like cells and the absence of pluripotent stem cells in differentiated EBs (Fig. 5). Staining showed that 5 day EBs formed from 500 and 2,000 cell cultures and harvested after 3 weeks of the differentiation process the number of cells expressing NANOG was lower than hESCs. Staining also confirmed SOX9 expression in the differentiated cells. ECM compounds characteristic of chondrocytes (such as type II collagen) were observed in the differentiated EBs. Moreover, we observed an expansive distribution pattern of chondroitin sulphate in these EBs in contrast to the consistent distribution observed in hESCs. Due to decreased cell motility and the high concentration of ECM deposition in large EBs after 15 days of suspension culture, we were unable to evaluate microscopically the specificity of immunofluorescence staining after 3 weeks differentiation process. Immunofluorescence staining was unable to determine the most prochondrogenic of the various differentiated EB populations. Overall, these results indicate the presence of chondrocyte-like cells and the differentiated cells were also heterogeneous.

\section{Discussion}

In our previous work we indicated that the EBs based differentiation protocol into chondrocyte-like cells is good, but not perfect $(15,22)$. For that purpose, we wanted to see if elongation of suspension culture would influence the chondrogenesis process. That is why the main aim of the present study was to evaluate the influence the number of cells in the culture on spontaneous and chondrogenic differentiation of EBs. The EB formation process was carried out in low-adhesion round bottom-well plates specifically designed for suspension culture in order to closely control the number of cells seeded in the wells and thus to obtain more homogenous EBs. After differentiation, most (approximately 78\%) of the EBs were homogenous in size and spherical. Although this is a relatively good outcome, published reports indicate lithographic well-design techniques can yield much higher rates of homogenous EBs (23-25). We also established the influence of 
A

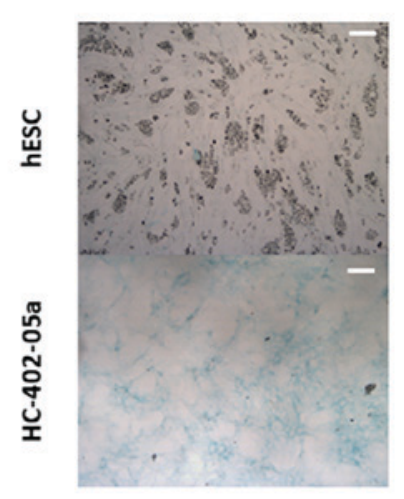

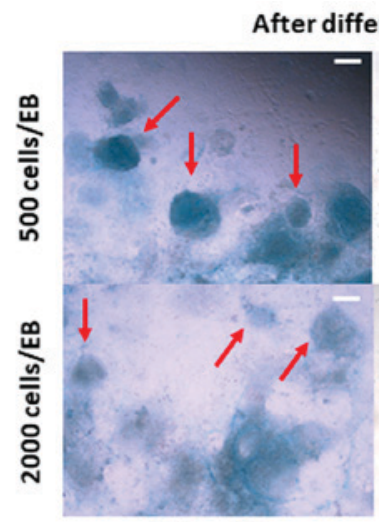

5 days EB

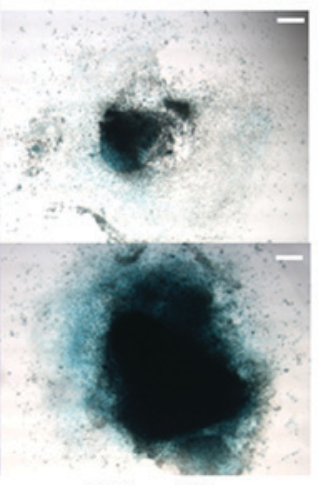

15 days EB
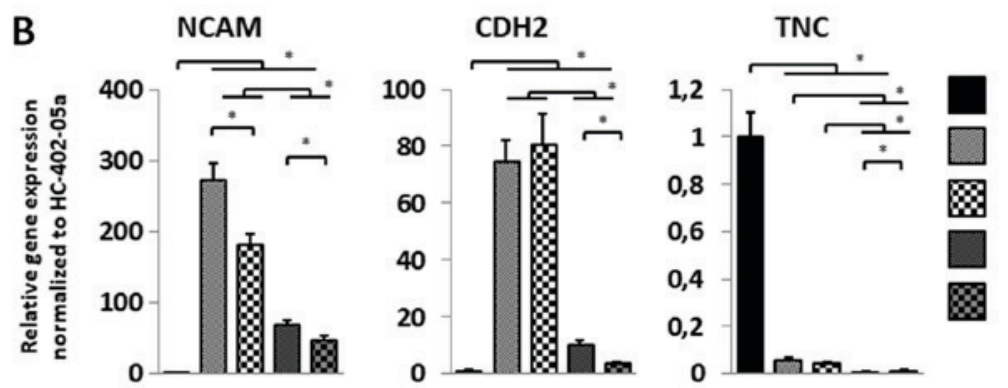

HC-402-05a

500 cells EB collected at $5^{\text {th }}$ day of culture

2000 cells EB collected at $5^{\text {th }}$ day of

culture

500 cells EB collected at $15^{\text {th }}$ day of

2000 cells EB collected at $15^{\text {th }}$ day of

culture

Figure 4. Cells differentiated from smaller EBs exhibit increased expression of genes associated with mesenchymal condensation and a more condensed cartilage nodule structure. (A) EBs harvested on day 5 of the suspension culture for chondrogenic differentiation presented more condensed cartilage-like nodules (red arrows). On day 15, the presence of intensively stained areas indicated a dense extracellular matrix in those structures. Scale bars, $200 \mu \mathrm{m}$. (B) To confirm mesenchymal condensation, reverse transcription-quantitative polymerase chain reaction of TNC, CDH2 and NCAM was performed. Mesenchymal condensation progressed more quickly in cells differentiated in 500-cell wells collected on day 5 of suspension culture. The y-axis represents the relative expression of analysed genes, normalized to the HC-402-05a cell line. Data are presented as the mean \pm standard deviation. "P<0,05, as indicated. EBs, embryoid bodies; hESC, human embryonic stem cells; TNC, tenascin C; CDH2, cadherin 2; NCAM, neural cell adhesion molecule.

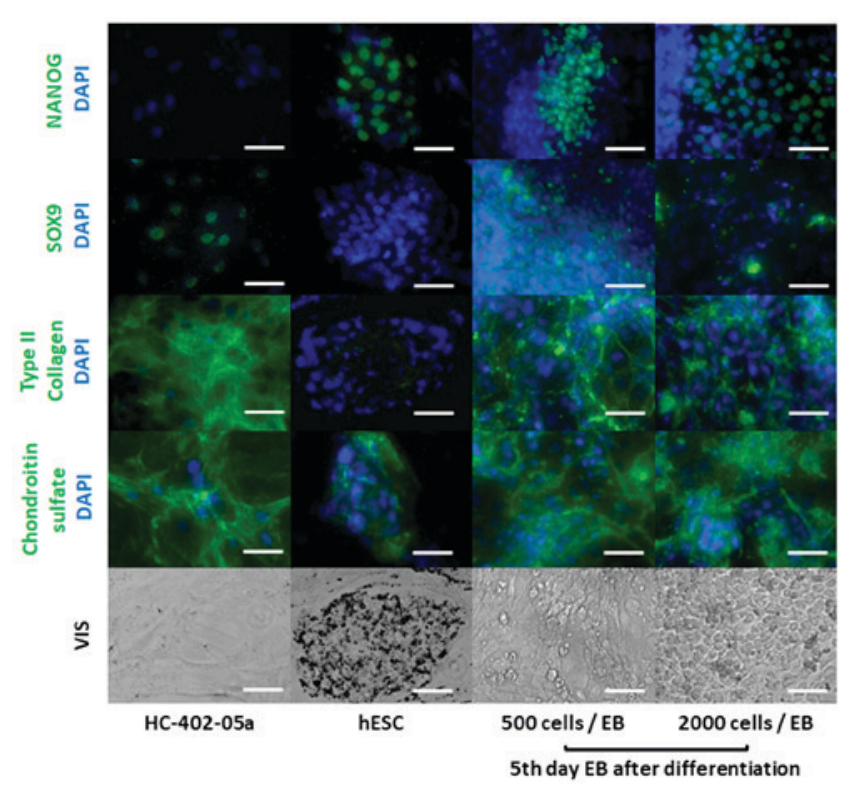

Figure 5. Chondrocyte-like and pluripotent cells were detected following the chondrogenic differentiation of EBs. Following 21 days of chondrogenic differentiation of EBs collected on the fifth or fifteenth day of suspension culture, the presence of cells exhibiting positive staining for NANOG was detected. Positively-stained SOX9 cells were also observed in the differentiated EBs. Additionally, extracellular matrix associated with chondrocytes such as type II collagen and chondroitin sulphate were detected, thus confirming successful chondrogenic differentiation. Magnification, x200; scale bars, $50 \mu \mathrm{m}$. EBs, embryoid bodies; hESC, human embryonic stem cells; SOX, sex determining region Y-box; VIS, visible light. varying cell quantities used for EB formation on spontaneous differentiation into three germ layers. Because the mesodermal germ layer is a chondrocyte precursor, we focused on the EBs that displayed the highest expression of mesoderm markers. We found that EBs formed in 500 and 1,000 cell cultures for 15 days expressed the mesodermal markers T and MIXL1 more highly than in other variants. $\mathrm{Ng}$ et al (26) showed that expression of the mesoderm germ layer markers in three hESC lines was higher in EBs formed in 500 and 1,000 cells. There is a strong correlation between EBs with a strong expression of mesoderm germ layer markers and the cell culture microenvironment. The global gene expression of EBs propagated in a standard suspension culture plate indicates a stronger upregulation of cardiac and mesoderm-related genes compared to ectoderm and endoderm genes, a phenomenon that can be explained by the diffusion of endogenous molecules secreted by EBs into the local microenvironment (27).

The results described above have prompted further research into the effect of the number of cells and the duration of suspension culture on chondrogenic differentiation. Based on the expression of Brachury and MIXL1, we decided to verify our hypothesis by differentiation of 500 cell wells EBs collected after 15 days in suspension culture. Additionally, we choose the 500 cell wells EBs after 5 days in suspension culture. This variant was chosen based on other studies revelations, where various progenitors derived from mesodermal germ layer where obtained more efficiently from younger and smaller EBs (26,28-30). Due to low expression of mesodermal markers as a variant with 
less prochondrogenic potential we choose 2,000 cell wells after 5th and 15th day of suspension culture. In our study, we estimated the expression of genes related to pluripotency and chondrogenesis after 21 days of chondrogenic differentiation. Unfortunately, the differentiated cells still expressed pluripotency markers (i.e., NANOG and SOX2). This is a common problem in all currently available differentiation techniques, and the continued expression of pluripotency markers even after completion of the differentiation process has been associated with an increased risk of teratomas (31). However, the use of oleic acid synthesis inhibitors, which is crucial for maintaining stemness and viability of pluripotent stem cells, could increase the clinical safety of differentiated cells. In our study, the differentiated EBs collected after 5 days in the suspension culture showed significant upregulation of $\mathrm{T}$ and FGFR3 compared to larger EBs and those collected after 15 days. SOX9 expression decreased compared to controls (articular chondrocyte cell line) but increased compared to EBs obtained from the other cell culture variants. Those results suggest that the chondrocyte-like cells obtained in this study were at the early stages of chondrogenesis. Chondrogenesis was confirmed by the upregulation of FGFR3 and COL2A1 in smaller EBs, which was correlated with the parallel upregulation of $\mathrm{T}$. This correlation is related to early limb formation, where $\mathrm{T}$ expression enhances recruitment of mesoderm cells towards chondroprogenitors via the FGFR3 pathway. Our findings with regards to the expression of molecules associated with ECM suggest chondrocytes in early stages of differentiation In addition, the expression of COL2A1, CD44, COL1A2 and ACAN decreased compared to controls, indicating an immature chondrocyte phenotype $(14,32)$. Another reason to explain why the obtained chondrocytes were premature is the behaviour of chondrocytes in native tissue, where ECM proteins are better expressed in three dimensional environments rather than in monolayer cultures. Other explanation for the premature chondrocytes includes culture conditions. We established the culture conditions in normoxia, which could also have negatively influenced the efficiency of chondrogenic differentiation given that hypoxic conditions are known to be more prochondrogenic. Similarly, the size of the EBs can also influence chondrogenic differentiation, as one study showed in EBs derived from mouse embryonic stem cells (28).

We also used alcian blue staining to evaluate the effect of EB mass on the deposition of proteoglycans, estimating the expression of genes related to mesenchymal condensation. In differentiated EBs, we observed blue stained areas, indicating the presence of deposited proteoglycans. EBs cultured for a longer time presented intensively stained areas, which could be interpreted as indicating more chondrogenic properties. Previous studies of the ECM composition of EBs have shown that the matrix is composed of laminins, type IV collagen, versican, fibronectin, hyaluronian, and/or type I collagen, which could explain the large, positively-stained areas of ECM in older EBs after differentiation $(33,34)$. As we expected, those findings could affect expression of genes related to the mesenchymal condensation process. Based on our results, we can make the following conclusions: first, the immaturity of the obtained chondrocytes was due to low TNC expression (35); second, the mesenchymal condensation process was more advanced in younger cells obtained from smaller (500 cell) cultures, which could be explained by higher NCAM levels.
The relation between $\mathrm{CDH} 2$ and NCAM during chondrogenesis could explain the lack of clear differences in $\mathrm{CDH} 2$ expression between EBs formed from 500 and 2,000 cell cultures and collected on day 5. Studies of the role of CDH2 and NCAM during in vitro chondrogenesis in chick limb buds have shown that $\mathrm{CDH} 2$ expression decreases during the finalization of chondrocyte maturation with a parallel increase in NCAM expression (36). The low expression of $\mathrm{CDH} 2$ in older differentiated EBs could explain the low cell motility, which was due to dense ECM observed in the intensively stained areas.

Immunofluorescence staining of the differentiated cells confirms the presence of NANOG, SOX9, type II collagen, and positively-stained chondroitin sulphate cells. Importantly, the pattern of chondroitin sulphate signalling in differentiated cells was similar to that observed in the chondrocyte cell line. Our results indicate the limitations of this approach: We obtained a mixed population of differentiated and undifferentiated cells, which is the main disadvantage of this technique. As mentioned previously, we did not passage or sort the cells during the experiments in order to achieve a broad overview of the influence of EB size on the chondrogenic process. This explains why we observed an increased accumulation of ECM in the EBs harvested on day 15, which did not allow us to estimate the influence of EB size and culture time on protein levels by immunofluorescence staining.

In this study, we have shown that several factors the duration of suspension culture, EB size, and particularly the number of plated cells used to form the EBs can significantly affect spontaneous differentiation and EB heterogeneity. In particular, we found that differentiated EBs formed from 500 cell cultures and collected on the 5th day of suspension culture presented better prochondrogenic properties. We determined that the decreased cellular mass of pluripotent stem cells used to form EBs positively affects chondrogenic differentiation and mesenchymal condensation. Differentiation of hESCs into chondrocytes with intermediate EBs generates chondrocyte-like cells at early stages of chondrogenesis. Therefore, additional steps are needed to obtain chondrocyte-like cells at more advanced stages of chondrogenesis, a finding that suggests that existing protocols will need to be modified.

\section{Acknowledgements}

The authors would like to thank Mr. Bradley Londres for his invaluable assistance in editing the final text.

\section{Funding}

The present study was funded by the National Science Centre allocated on the basis of (grant no. 2012/07/E/NZ3/01819).

\section{Availability of data and material}

All data generated or analysed during this study are included in this published article.

\section{Authors' contributions}

WMS and TT were responsible for experimental design. KK and KJ performed the experiments. WMS, MSL, KK, MR and KJ 
analysed and interpreted the results. All authors were responsible for the writing and preparation of the manuscript and figures.

\section{Ethics approval and consent to participate}

Not applicable.

\section{Patient consent for publication}

Not applicable.

\section{Competing interests}

The authors declare that they have no competing interests.

\section{References}

1. de Windt TS, Vonk LA, Brittberg M and Saris DBF: Treatment and prevention of (Early) osteoarthritis using articular cartilage repair-fact or fiction? A systematic review. Cartilage 4 (3 Suppl): 5S-12S, 2013.

2. Li Y, Wei X, Zhou J and Wei L: The age-related changes in cartilage and osteoarthritis. Biomed Res Int 2013: 916530, 2013.

3. Madry H, Luyten FP and Facchini A: Biological aspects of early osteoarthritis. Knee Surg Sports Traumatol Arthrosc 20 407-422, 2012.

4. Moreira Teixeira LS, Leijten JC, Sobral J, Jin R, van Apeldoorn AA, Feijen J, van Blitterswijk C, Dijkstra PJ and Karperien M: High throughput generated micro-aggregates of chondrocytes stimulate cartilage formation in vitro and in vivo. Eur Cell Mater 23: 387-399, 2012

5. Caron MM,EmansPJ,Coolsen MM, VossL, SurtelDA,Cremers A, van Rhijn LW and Welting TJ: Redifferentiation of dedifferentiated human articular chondrocytes: Comparison of 2D and 3D cultures. Osteoarthritis Cartilage 20: 1170-1178, 2012

6. Carroll SH and Ravid K: Differentiation of mesenchymal stem cells to osteoblasts and chondrocytes: A focus on adenosine receptors. Expert Rev Mol Med 15: e1, 2013.

7. Solchaga LA, Penick KJ and Welter JF: Chondrogenic differentiation of bone marrow-derived mesenchymal stem cells: Tips and tricks. In: Methods in molecular biology. Vol 698. Vemuri M, Chase LG and Rao MS (eds.) Humana Press, Totowa, NJ, pp253-278, 2011.

8. Kanawa M, Igarashi A, Ronald VS, Higashi Y, Kurihara H, Sugiyama M, Saskianti T, Pan H and Kato Y: Age-dependent decrease in the chondrogenic potential of human bone marrow mesenchymal stromal cells expanded with fibroblast growth factor-2. Cytotherapy 15: 1062-1072, 2013.

9. Ko JY, Kim KI, Park S and Im GI: In vitro chondrogenesis and in vivo repair of osteochondral defect with human induced pluripotent stem cells. Biomaterials 35: 3571-3581, 2014.

10. Tsumaki N, Okada M and Yamashita A: iPS cell technologies and cartilage regeneration. Bone 70: 48-54, 2015.

11. Robinton DA and Daley GQ: The promise of induced pluripotent stem cells in research and therapy. Nature 481: 295-305, 2012.

12. Beers J, Linask KL, Chen JA, Siniscalchi LI, Lin Y, Zheng W, Rao M and Chen G: A cost-effective and efficient reprogramming platform for large-scale production of integration-free human induced pluripotent stem cells in chemically defined culture. Sci Rep 5: 11319, 2015.

13. Toh WS, Guo XM,Choo AB,Lu K,LeeEH and Cao T: Differentiation and enrichment of expandable chondrogenic cells from human embryonic stem cells in vitro. J Cell Mol Med 13: 3570-3590, 2009.

14. Oldershaw RA, Baxter MA, Lowe ET, Bates N, Grady LM, Soncin F, Brison DR, Hardingham TE and Kimber SJ: Directed differentiation of human embryonic stem cells toward chondrocytes. Nat Biotechnol 28: 1187-1194, 2010.

15. Suchorska WM, Augustyniak E, Richter M and Trzeciak T: Comparison of four protocols to generate chondrocyte-like cells from human induced pluripotent stem cells (hiPSCs). Stem Cell Rev 13: 299-308, 2017.

16. Mahmoudifar N and Doran PM: Chondrogenesis and cartilage tissue engineering: The longer road to technology development Trends Biotechnol 30: 166-176, 2012.
17. Moon SH, Ju J, Park SJ, Bae D, Chung HM and Lee SH: Optimizing human embryonic stem cells differentiation efficiency by screening size-tunable homogenous embryoid bodies. Biomaterials 35: 5987-5997, 2014.

18. Preda MB, Burlacu A and Simionescu M: Defined-size embryoid bodies formed in the presence of serum replacement increases the efficiency of the cardiac differentiation of mouse embryonic stem cells. Tissue Cell 45: 54-60, 2013.

19. Van Winkle AP, Gates ID and Kallos MS: Mass transfer limitations in embryoid bodies during human embryonic stem cell differentiation. Cells Tissues Organs 196: 34-47, 2012.

20. Hwang YS, Chung BG, Ortmann D, Hattori N, Moeller HC and Khademhosseini A: Microwell-mediated control of embryoid body size regulates embryonic stem cell fate via differential expression of WNT5a and WNT11. Proc Natl Acad Sci USA 106: 16978-16983, 2009.

21. Livak KJ and Schmittgen TD: Analysis of relative gene expression data using real-time quantitative PCR and the 2(-Delta Delta C(T)) method. Methods 25: 402-408, 2001.

22. Suchorska WM, Lach MS, Richter M, Kaczmarczyk J and Trzeciak T: Bioimaging: An useful tool to monitor differentiation of human embryonic stem cells into chondrocytes. Ann Biomed Eng 44: 1845-1859, 2016.

23. Dias AD, Unser AM, Xie Y, Chrisey DB and Corr DT: Generating size-controlled embryoid bodies using laser direct-write. Biofabrication 6: 025007, 2014.

24. Xu F, Sridharan B, Wang S, Gurkan UA, Syverud B and Demirci U: Embryonic stem cell bioprinting for uniform and controlled size embryoid body formation. Biomicrofluidics 5: 22207, 2011

25. Pettinato G, Wen X and Zhang N: Formation of well-defined embryoid bodies from dissociated human induced pluripotent stem cells using microfabricated cell-repellent microwell arrays. Sci Rep 4: 7402, 2014.

26. Ng ES, Davis RP, Azzola L, Stanley EG and Elefanty AG: Forced aggregation of defined numbers of human embryonic stem cells into embryoid bodies fosters robust, reproducible hematopoietic differentiation. Blood 106: 1601-1603, 2005.

27. Giobbe GG, Zagallo M, Riello M, Serena E, Masi G, Barzon L, Di Camillo B and Elvassore N: Confined 3D microenvironment regulates early differentiation in human pluripotent stem cells. Biotechnol Bioeng 109: 3119-3132, 2012.

28. Messana JM, Hwang NS, Coburn J, Elisseeff JH and Zhang Z: Size of the embryoid body influences chondrogenesis of mouse embryonic stem cells. J Tissue Eng Regen Med 2: 499-506, 2008.

29. Cha JM, Bae H, Sadr N, Manoucheri S, Edalat F, Kim K, Kim SB, Kwon IK, Hwang YS and Khademhosseini A: Embryoid body size-mediated differential endodermal and mesodermal differentiation using polyethylene glycol (PEG) Microwell Array. Mac Res 23: 245-255, 2015.

30. Lee EJ, Lee HN, Kang HJ, Kim KH, Hur J, Cho HJ, Lee J, Chung HM, Cho J, Cho MY, et al: Novel embryoid body-based method to derive mesenchymal stem cells from human embryonic stem cells. Tissue Eng Part A 16: 705-715, 2010.

31. Cheng A, Kapacee Z, Peng J, Lu S, Lucas RJ, Hardingham TE and Kimber SJ: Cartilage repair using human embryonic stem cell-derived chondroprogenitors. Stem Cells Transl Med 3: 1287-1294, 2014.

32. Yang SL, Harnish E, Leeuw T, Dietz U, Batchelder E, Wright PS, Peppard J, August P, Volle-Challier C, Bono F, et al: Compound screening platform using human induced pluripotent stem cells to identify small molecules that promote chondrogenesis. Protein Cell 3: 934-942, 2012.

33. Goh SK, Olsen P and Banerjee I: Extracellular matrix aggregates from differentiating embryoid bodies as a scaffold to support ESC proliferation and differentiation. PLoS One 8: e61856, 2013

34. Shukla S, Nair R, Rolle MW, Braun KR, Chan CK, Johnson PY, Wight TN and McDevitt TC: Synthesis and organization of hyaluronan and versican by embryonic stem cells undergoing embryoid body differentiation. J Histochem Cytochem 58: 345-358, 2010.

35. Okamura N, Hasegawa M, Nakoshi Y, Iino T, Sudo A, Imanaka-Yoshida K, Yoshida T and Uchida A: Deficiency of tenascin-C delays articular cartilage repair in mice. Osteoarthritis Cartilage 18: 839-848, 2010.

36. Tavella S, Raffo P, Tacchetti C, Cancedda R and Castagnola P: $\mathrm{N}-\mathrm{CAM}$ and N-cadherin expression during in vitro chondrogenesis. Exp Cell Res 215: 354-362, 1994. 